\title{
Collider Physics and Cosmology
}

\author{
Jonathan L. Feng \\ Department of Physics and Astronomy, University of California, Irvine, CA 92697, \\ USA
}

\begin{abstract}
In the coming year, the Large Hadron Collider will begin colliding protons at energies nearly an order of magnitude beyond the current frontier. The LHC will, of course, provide unprecedented opportunities to discover new particle physics. Less well-known, however, is that the LHC may also provide insights about gravity and the early universe. I review some of these connections, focusing on the topics of dark matter and dark energy, and highlight outstanding prospects for breakthroughs at the interface of particle physics and cosmology.

PACS numbers: 13.85.-t, 95.35.+d, 95.36.+x
\end{abstract}

\section{Introduction}

The Large Hadron Collider (LHC) is scheduled to begin running in the summer of 2008. Conceived around 1984 and approved in 1994, the LHC will provide the first detailed look at the weak energy scale $M_{\text {weak }} \sim 100 \mathrm{GeV}-1 \mathrm{TeV}$ by colliding protons with protons at the center-of-mass energy $E_{\mathrm{CM}}=14 \mathrm{TeV}$ and ultimate luminosity $\mathcal{L}=100 \mathrm{fb}^{-1} / \mathrm{yr}$. This is far beyond the current energy frontier, where the Tevatron collides protons and anti-protons with $E_{\mathrm{CM}}=2 \mathrm{TeV}$ and $\mathcal{L} \sim 1 \mathrm{fb}^{-1} / \mathrm{yr}$. As an illustration of the power of the LHC, top quarks, discovered in 1994 with a handful of events and currently produced at the Tevatron at the rate of $\sim 1000$ per year, will be produced at the rate of $10 \mathrm{~Hz}$ when the LHC reaches its design luminosity.

The raison d'etre for the LHC is the discovery of the Higgs boson and associated microphysics, including supersymmetric and other postulated particles. In recent years, however, the LHC's potential for providing insights into gravity and cosmology have taken on increasing importance. My goal here is to review some recent developments and to highlight a few scenarios in which the implications of the LHC for our understanding of gravity and the early universe may, in fact, be profound.

\section{Cosmology Now}

A wealth of recent cosmological observations now constrain the total energy densities of non-baryonic dark matter, dark energy, and baryons to be [1, 2]

$$
\Omega_{\mathrm{DM}}=0.23 \pm 0.044
$$




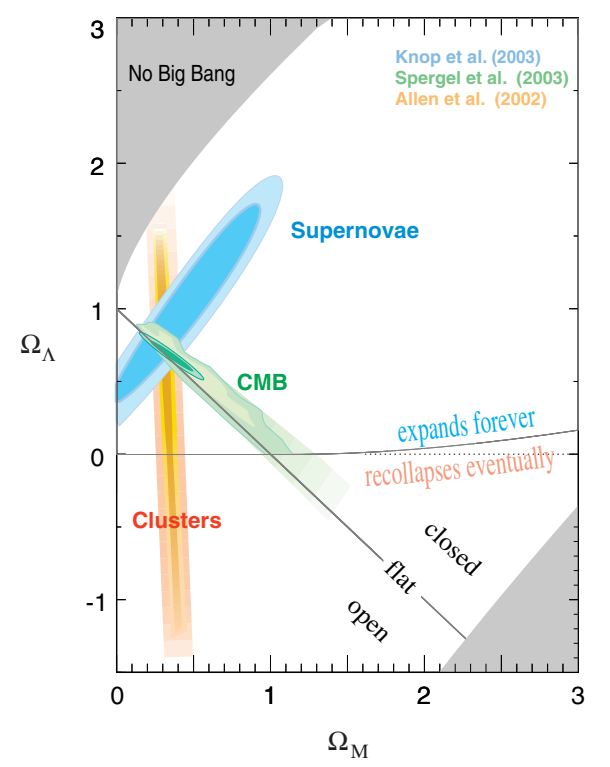

Figure 1. Constraints on $\Omega_{\mathrm{M}}$ and $\Omega_{\Lambda}$ from observations of supernovae, the CMB, and galaxy clusters. Boundaries between regions with open and closed universes, between universes that expand forever and those that recollapse, and regions that do not extrapolate back to a Big Bang singlularity, are also shown [3].

$$
\begin{aligned}
& \Omega_{\Lambda}=0.73 \pm 0.04 \\
& \Omega_{B}=0.04 \pm 0.004
\end{aligned}
$$

The constraints are summarized in Fig. 1 and both the central values and the error bars are remarkable. Given that just a decade ago the range $0.2 \lesssim \Omega_{\mathrm{DM}} \lesssim 0.6$ was allowed and $\Omega_{\Lambda}=0$ was often assumed, this represents spectacular progress.

At the same time, this progress highlights many outstanding questions. These include:

- Dark matter: What is its mass? What are its spin and other quantum numbers? Is it absolutely stable? What is the symmetry origin of the dark matter particle? Is dark matter composed of one particle species or many? How and when was it produced? Why does $\Omega_{\mathrm{DM}}$ have the observed value? What was its role in structure formation? How is dark matter distributed now?

- Dark energy: What is it? Why is $\Omega_{\Lambda}$ not much larger than observed? Why not $\Omega_{\Lambda}=0$ ? Does it evolve?

- Baryons: Why not $\Omega_{B}=0$ ? Is this related to neutrinos and leptonic CP violation? Where are all the baryons?

Although colliders may also shed light on baryogenesis, I will focus here on dark matter and dark energy. From a microphysical viewpoint, these problems appear, at least at first, to be completely different. In the case of dark matter, no known particles contribute, there are reasons to believe that the problem is tied to the weak energy scale $M_{\text {weak }}$, and there are several compelling candidates. In contrast, for dark energy, 
all known particles contribute through their zero-point energy, the problem appears to be intrinsically tied to the Planck scale $M_{\mathrm{Pl}} \sim 10^{19} \mathrm{GeV}$, and there are no compelling solutions.

In the following sections, I will discuss dark matter and dark energy in turn, focusing primarily on dark matter, where the collider connections to cosmology and gravity are especially concrete and compelling.

\section{Dark Matter}

\subsection{The "WIMP Miracle"}

The particle or particles that make up most of dark matter must be

- stable, or at least long-lived on cosmological time scales,

- cold or warm to properly seed structure formation, and

- non-baryonic, to preserve the successes of Big Bang nucleosynthesis (BBN).

Unfortunately, these constraints are no match for the creativity of theorists, who have proposed scores of viable candidates with masses and interaction cross sections varying over tens of orders of magnitude.

Candidates with masses at the weak scale $M_{\text {weak }} \sim 100 \mathrm{GeV}-1 \mathrm{TeV}$ have received much of the attention, however. There are several good reasons for this. First, new particles at the weak scale are independently motivated by attempts to understand the Higgs boson and electroweak symmetry breaking. In the standard model of particle physics, the Higgs boson's mass is naturally raised by radiative corrections to be far above $M_{\text {weak }}$. This conflicts with precision measurements, which constrain the Higgs mass to $m_{h} \sim M_{\text {weak }}$. This puzzle is the gauge hierarchy problem. New ideas are required to resolve this problem, and these ideas invariably predict new particle states with masses around $M_{\text {weak }}$.

In addition, although we have not seen any of these new particles, there are already indications that if these particles exist, they are stable. This is the cosmological legacy of LEP, the Large Electron-Positron Collider that ran from 1989-2000. Generically, the new particles introduced to solve the gauge hierarchy problem induce new interactions $(\mathrm{SM})(\mathrm{SM}) \rightarrow \mathrm{NP} \rightarrow(\mathrm{SM})(\mathrm{SM})$, where $\mathrm{SM}$ and $\mathrm{NP}$ denote standard model and new particles, respectively. LEP, along with the Stanford Linear Collider, looked for the effects of these interactions and found none. At the same time, the new particles cannot be decoupled completely; to solve the gauge hierarchy problem, they must interact with the Higgs boson through couplings $h \leftrightarrow(\mathrm{NP})(\mathrm{NP})$, and they cannot be too heavy. A simple and elegant solution is to require a conserved discrete parity that requires all interactions to involve an even number of new particles [4, 5]. As a side effect, this discrete parity implies that the lightest new particle cannot decay - it is stable, as required for dark matter.

Finally, if these new particles exist and are stable, they are naturally produced with the cosmological densities required of dark matter. This fact is sometimes called the 
"WIMP miracle," and it is particularly tantalizing. Dark matter may be produced in a simple and predictive manner as a thermal relic of the Big Bang. The evolution of a thermal relic's number density is shown in Fig. 2. In stage (1), the early Universe is dense and hot, and all particles are in thermal (chemical) equilibrium. In stage (2), the Universe cools to temperatures $T$ below the dark matter particle's mass $m_{\chi}$, and the number of dark matter particles becomes Boltzmann suppressed, dropping exponentially as $e^{-m_{\chi} / T}$. In stage (3), the Universe becomes so cool and dilute that the dark matter annihilation rate is too low to maintain equilibrium. The dark matter particles then "freeze out," with their number asymptotically approaching a constant, their thermal relic density.

More detailed analysis shows that the thermal relic density is rather insensitive to $m_{\chi}$ and inversely proportional to the annihilation cross section:

$$
\Omega_{\mathrm{DM}} \sim\left\langle\sigma_{A} v\right\rangle^{-1},
$$

where $v$ is the relative velocity of the annihilating particles, and the brackets indicate a thermal average. The constant of proportionality depends on the details of the microphysics, but we may derive a rough estimate. On dimensional grounds, the cross section can be written

$$
\sigma_{A} v=k \frac{4 \pi \alpha_{1}^{2}}{m_{\chi}^{2}}\left(1 \text { or } v^{2}\right),
$$

where the factor $v^{2}$ is absent or present for $S$ - or $P$-wave annihilation, respectively, and terms higher-order in $v$ have been neglected. The constant $\alpha_{1}$ is the hypercharge fine structure constant, related to the weak interactions of the standard model, and $k$ parameterizes deviations from this estimate.

With this parametrization, given a choice of $k$, the relic density is determined as a function of $m_{\chi}$. The results are shown in Fig. 2. The width of the band comes from considering both $S$ - and $P$-wave annihilation, and from letting $k$ vary from $\frac{1}{2}$ to 2 . We see that a particle that makes up all of dark matter is predicted to have mass in the range $m_{\chi} \sim 100 \mathrm{GeV}-1 \mathrm{TeV}$; a particle that makes up $10 \%$ of dark matter, still significant with respect to its impact on structure formation, for example, has mass $m_{\chi} \sim 30 \mathrm{GeV}-300 \mathrm{GeV}$. There are models in which the effective $k$ is outside our illustrative range. In fact, values of $k$ smaller than we have assumed, predicting smaller $m_{\chi}$, are not uncommon, as the masses of virtual particles in annihilation diagrams can be significantly higher than $m_{\chi}$. However, the general conclusion remains: particles that interact through weak interactions and have mass at the weak scale naturally have significant thermal relic densities.

To summarize, viable particle physics theories designed to address the gauge hierarchy problem naturally (1) predict new weakly-interacting particles with mass $\sim M_{\text {weak }}$ that (2) are stable and (3) have the thermal relic densities required to be dark matter. The convergence of particle physics and cosmological requirements for new states of matter has motivated many new proposals for dark matter. These may 

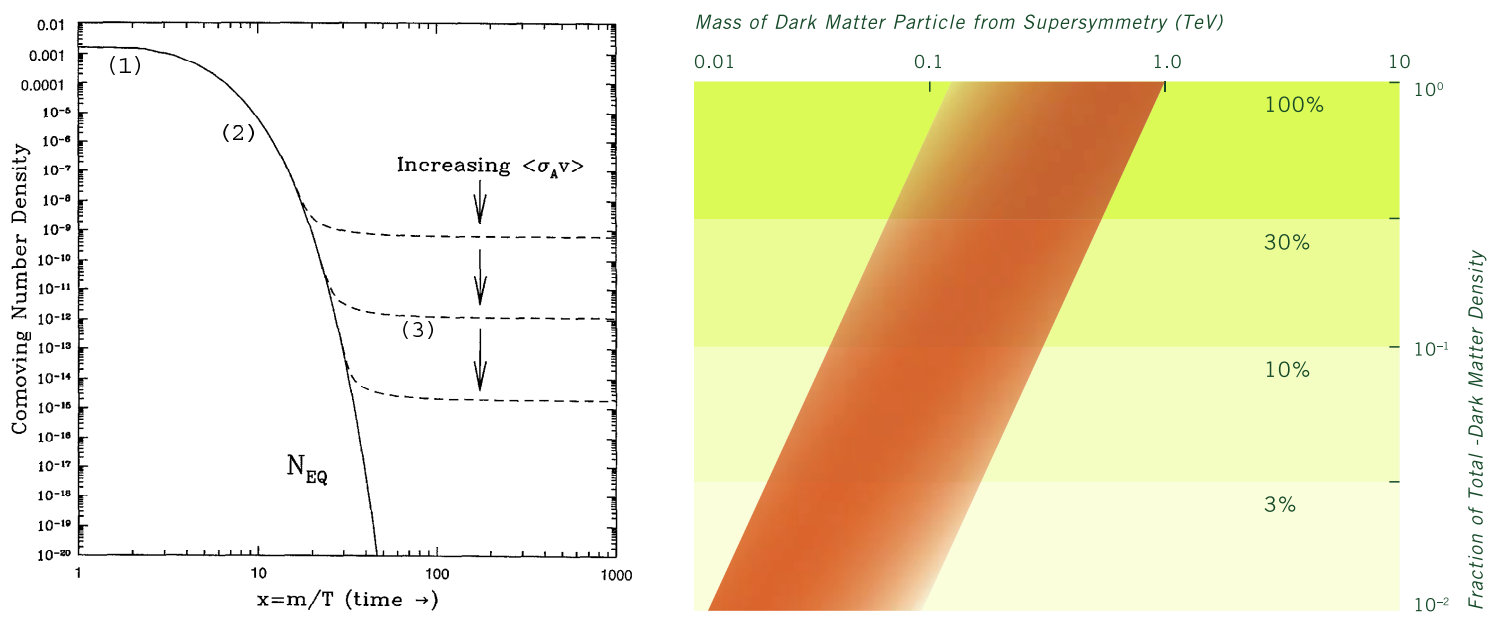

Figure 2. Left: The cosmological evolution of a thermal relic's comoving number density [6]. Right: A band of natural values in the $\left(m_{\chi}, \Omega_{\chi}\right)$ plane for a thermal relic [7].

be grouped into two classes: WIMPs and superWIMPs. In the following subsections, we consider what insights colliders may provide in each of these two cases.

\subsection{WIMPs}

WIMPs, weakly-interacting massive particles, interact through the weak force and have masses near the weak scale $M_{\text {weak }}$. For the reasons given above, the WIMP paradigm is now thriving, and recent years have seen a proliferation of WIMP candidates. These include the traditional prototype, neutralinos in supersymmetry with $R$-parity conservation [8, 9], but also more recent candidates, including Kaluza-Klein photons in universal extra dimensions with KK-parity [10, 11], branons in brane world scenarios with branon parity [12, 13], and T-odd dark matter in little Higgs models with $T$ parity [5].

If WIMPs are the dark matter, what can colliders tell us? This has been investigated in numerous studies [14]. Given the energy of the LHC and the requirement that WIMPs interact through the weak force, WIMPs will almost certainly be produced in large numbers at the LHC, but their detection will be somewhat indirect. For example, in supersymmetry, the LHC will typically produce pairs of squarks and gluinos. These will then decay through some cascade chain, eventually ending up in neutralino WIMPs, which escape the detector. Their existence is registered only through the signature of missing energy and momentum. Although the observation of missing particles is consistent with the production of dark matter in the lab, it is far from compelling evidence. In particular, colliders can only establish that the neutralino was stable enough to exit the detector, typically implying that the neutralino's lifetime was $\tau>10^{-7} \mathrm{~s}$, a far cry from the criterion $\tau \gtrsim 10^{17}$ s required for dark matter. Supersymmetric scenarios are not special in this regard - although not examined in as much detail to date, other WIMP models, such as those derived from universal extra dimensions [15], share all of 
the features and caveats noted above for supersymmetry.

Clearly more is needed. In the last few years, there has been a great deal of progress in this direction. The main point of this progress has been to show that colliders can perform detailed studies of new physics, and this can constrain the dark matter candidate's properties so strongly that the candidate's thermal relic density can be precisely determined. The consistency of this density with the cosmologically observed density would then be strong evidence that the particle produced at colliders is, in fact, the cosmological dark matter.

This approach is analogous to the well-known case of BBN. For BBN, data from nuclear physics experiments stringently constrain cross sections involving the light nuclei. Along with the assumption of a cooling and expanding universe, this allows one to predict the light element abundances left over from the Big Bang. These predictions can be compared to observation, and their consistency gives us confidence that the light elements were actually created in this way.

For dark matter, the idea is that particle physics experiments at the LHC may stringently constrain cross sections involving dark matter and related particles. Along with the assumption of a cooling and expanding universe, this microscopic data allows one to predict the dark matter relic density, basically by following the relic density curves of Fig. 2. This thermal relic density may be compared to the observed density of dark matter, and their consistency would give us confidence that dark matter is actually produced in this way and is made of the particles produced at the collider.

Although the plan is simple, carrying it out is far from straightforward. As with the case of BBN, where thousands of nuclear processes enter the picture, there are many particle physics processes that contribute to the dark matter annihilation cross section $\sigma_{A}$ of Eq. (4). In the case of supersymmetry, for example, the relevant processes are given in Fig. 3. The task at a collider is to determine the masses and couplings of all the new particles entering these processes, or to bound them sufficiently to ensure that their contributions are negligible.

How well can the LHC do? The answer depends sensitively on the underlying dark matter scenario, but several qualitatively different cases have now been studied [16, 17, 18, 19. The results of one (admittedly rather exemplary) supersymmetric case study are given in Fig. 4. In conjunction with other cosmological observations, the WMAP satellite constrains the dark matter relic density $\Omega_{\chi}$ to a fractional uncertainty of $\pm 6 \%$. Its successor, Planck, is expected to sharpen this to $\pm 2 \%$. At the same time, precision studies at the LHC can determine so many of the supersymmetric model parameters that the neutralino thermal relic density can be predicted to $\pm 20 \%$. Measurements at the International Linear Collider, a proposed $e^{+} e^{-}$collider, could improve this to $\pm 3 \%$.

Consistency between the particle physics predictions and the cosmological observations would provide compelling evidence that the particle produced at the LHC is in fact dark matter. Of course, the colliders also determine many other properties of the dark matter along the way; the mass can be highly constrained, as shown, as can its spin and many other properties. In this way, colliders may finally help solve 


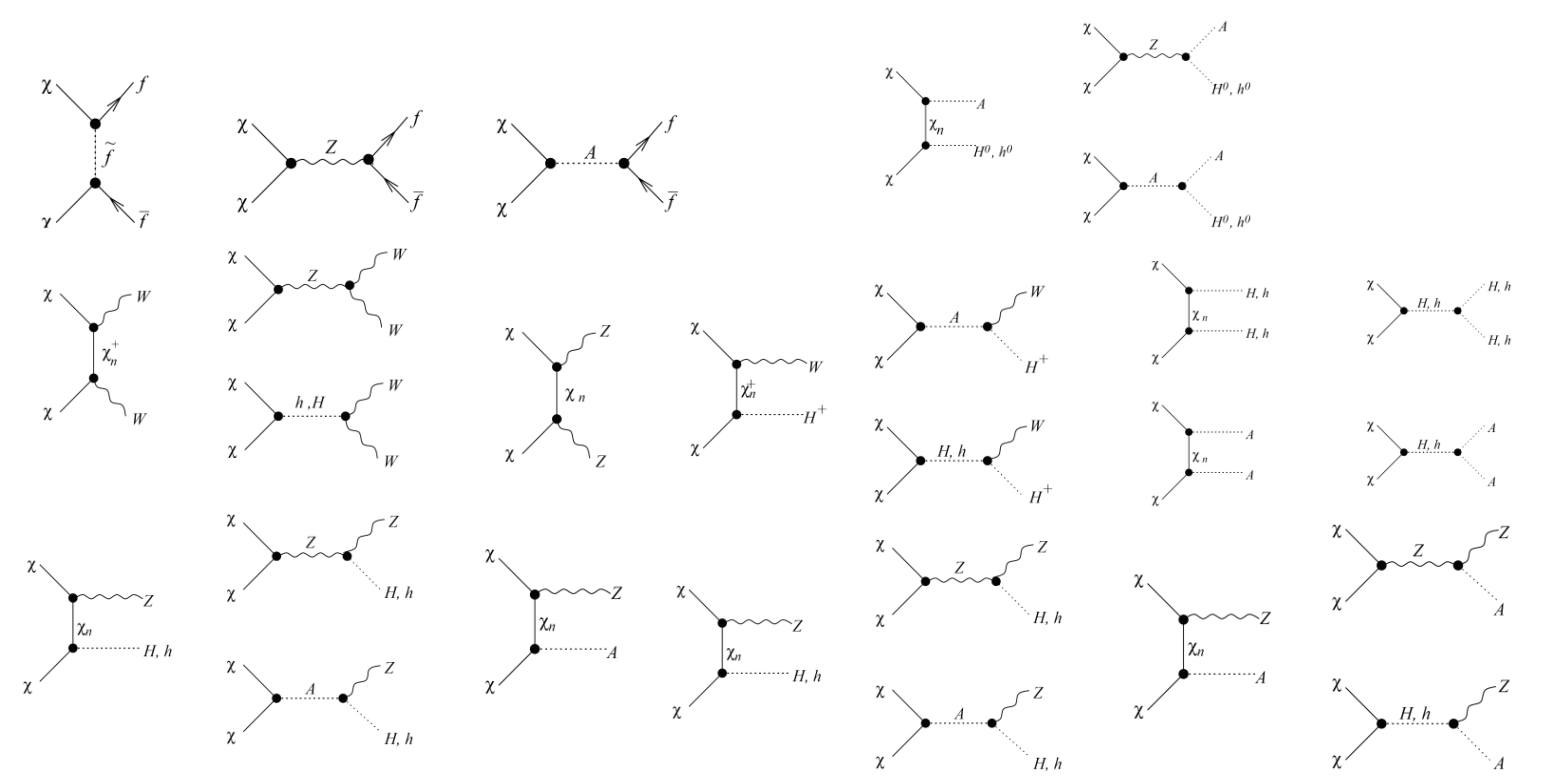

Figure 3. Particle physics processes that contribute to the annihilation cross section for $\chi \chi \rightarrow$ anything, where $\chi$ is the neutralino dark matter of supersymmetry [6].

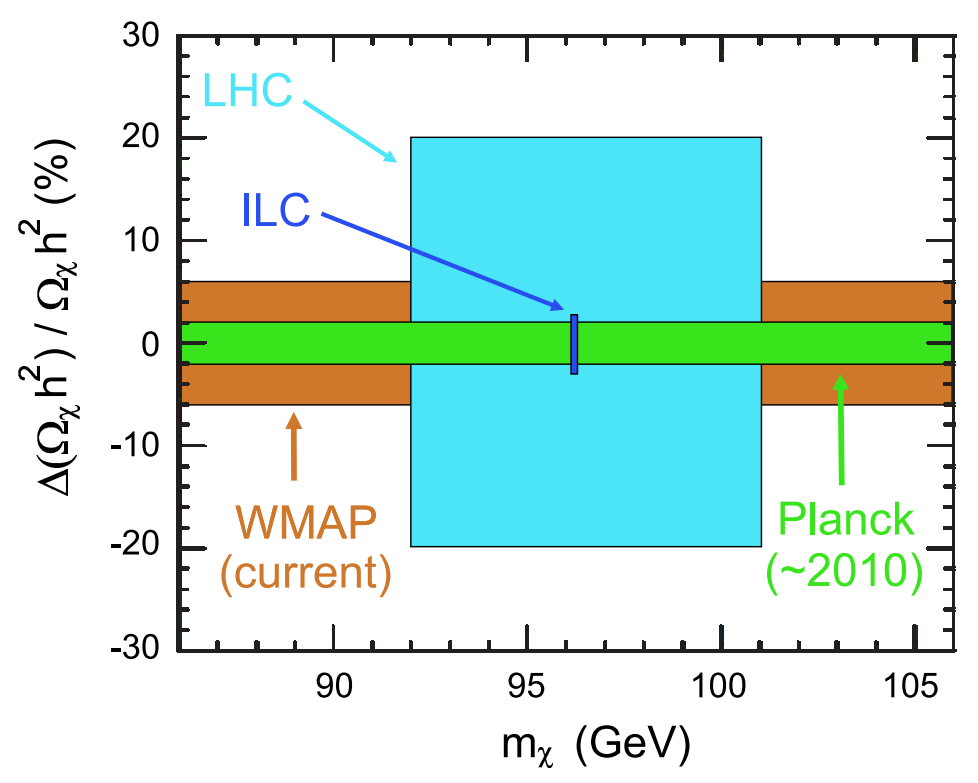

Figure 4. Constraints in the $\left(m_{\chi}, \Delta\left(\Omega_{\chi} h^{2}\right) / \Omega_{\chi} h^{2}\right)$ plane from the LHC and ILC, and from the WMAP and Planck satellite experiments [20. The satellite experiments measure $\Omega_{\chi}$, but are insensitive to the dark matter mass $m_{\chi}$; the collider experiments bound both.

the question of the microscopic identity of dark matter. Note also that, just as BBN gives us confidence that we understand the universe back to times of 1 second after the Big Bang and temperatures of $1 \mathrm{MeV}$, such studies also provide a window on the era of dark matter freezeout, or roughly times of 1 nanosecond after the Big Bang, and temperatures of $\sim 10 \mathrm{GeV}$.

Of course, the thermal relic density prediction from colliders and the cosmological 


\section{IDENTIFYING DARK MATTER}

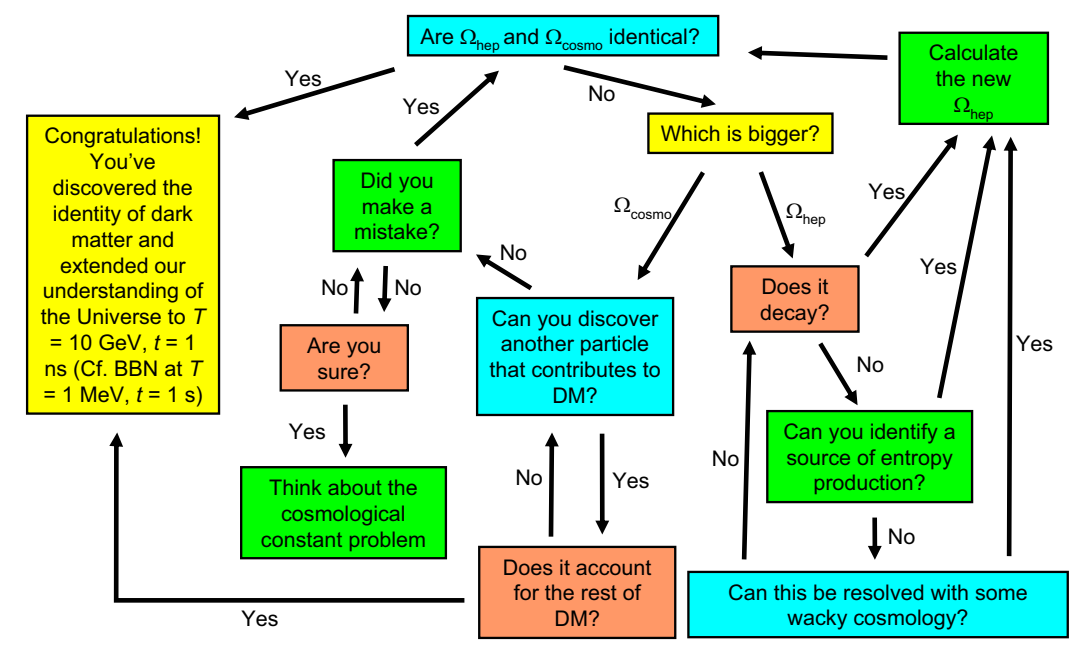

Figure 5. Flowchart illustrating the possible implications of comparing $\Omega_{\mathrm{hep}}$, the predicted dark matter thermal relic density determined from high energy physics, and $\Omega_{\text {cosmo, }}$, the actual dark matter relic density determined by cosmological observations.

observations need not be consistent. In this case, there are many possible lines of inquiry, depending on which is larger. A flowchart of possibilities is given in Fig. 5 ,

\subsection{SuperWIMPs}

Strictly speaking, dark matter need only be gravitationally interacting - there is as yet no evidence of any other sort of interaction. However, the "WIMP miracle" described in Sec. 3.1 might appear to require that dark matter have weak interactions if its relic density is naturally to be in the right range. This is not true, however - dark matter may be composed of superweakly-interacting massive particles, superWIMPs, which have interactions weaker than weak, but still naturally have the required relic density.

In superWIMP scenarios [21], a WIMP freezes out as usual, but then decays to a superWIMP, as shown in Fig. 6. As with WIMPs, there has recently been a proliferation of superWIMP candidates. The prototypical example of a superWIMP is a weak-scale gravitino produced non-thermally in the late decays of a supersymmetric WIMP, such as a neutralino, charged slepton, or sneutrino [21, 22, 23, 24]. Additional examples include axinos [25] and quintessinos [26] in supersymmetry, Kaluza-Klein graviton and axion states in models with universal extra dimensions [27], and stable particles in models that simultaneously address the problem of baryon asymmetry [28]. SuperWIMPs have all of the virtues of WIMPs. They exist in the same well-motivated frameworks and are stable for the same reasons. In addition, in the natural case that the decaying WIMP and superWIMP have comparable masses, superWIMPs also are naturally produced with relic densities of the desired order of magnitude.

Collider evidence for superWIMPs may come in one of two forms. Collider 


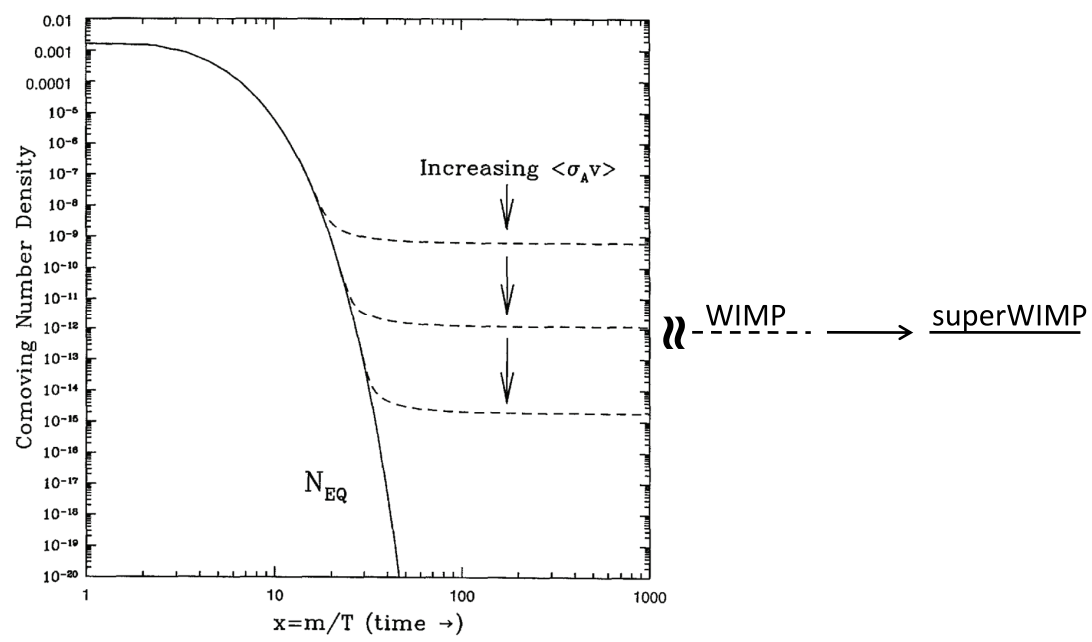

Figure 6. In superWIMP scenarios, a WIMP freezes out as usual, but then decays to a superWIMP, a superweakly-interacting particle that forms dark matter.

experiments may find evidence for charged, long-lived particles. Given the stringent bounds on charged dark matter, such particles presumably decay, and their decay products may be superWIMPs. Alternatively, colliders may find seemingly stable WIMPs, but the WIMP relic density studies described in Sec. 3.2 may favor a relic density that is too large, providing evidence that WIMPs decay. These two possibilities are not mutually exclusive. In fact, the discovery of charged long-lived particles with toolarge predicted relic density is a distinct possibility and would provide strong motivation for superWIMP dark matter.

Because superWIMPs are produced in the late decays of WIMPs, their number density is therefore identical to the WIMP number density at freeze out, and the superWIMP relic density is

$$
\Omega_{\mathrm{sWIMP}}=\frac{m_{\mathrm{sWIMP}}}{m_{\mathrm{WIMP}}} \Omega_{\mathrm{WIMP}} .
$$

To determine the superWIMP relic density, we must therefore determine the superWIMP's mass. This is not easy, since the WIMP lifetime may be very large, implying that superWIMPs are typically produced long after the WIMPs have escaped collider detectors. As an example, consider the case of supersymmetry with a stau next-to-lightest supersymmetric particle (NLSP) decaying to a gravitino superWIMP. Gravitinos interact only gravitationally, and so this decay is suppressed by Newton's constant $G_{N}$. On dimensional grounds, we therefore expect the stau lifetime to be $1 /\left(G_{N} M_{\text {weak }}^{3}\right)$. More precisely, we find

$$
\tau(\tilde{\tau} \rightarrow \tau \tilde{G})=\frac{6}{G_{N}} \frac{m_{\tilde{G}}^{2}}{m_{\tilde{\tau}}^{5}}\left[1-\frac{m_{\tilde{G}}^{2}}{m_{\tilde{\tau}}^{2}}\right]^{-4} \sim 10^{4}-10^{8} \mathrm{~s} .
$$

This is outlandishly long by particle physics standards. This gravitino superWIMP scenario therefore implies that the signal of supersymmetry at colliders will be metastable sleptons with lifetimes of days to months. Such particles will produce slowlymoving particles that should be obvious at the LHC [29, 30, 31, 32. 


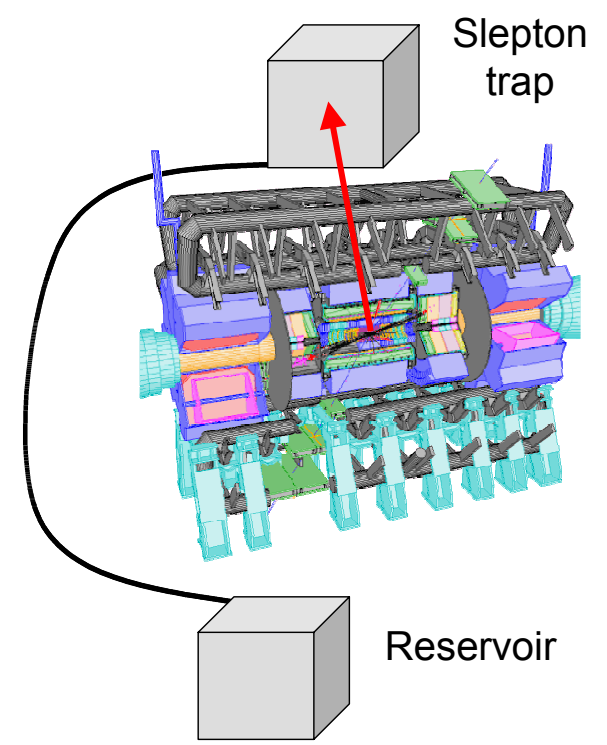

Figure 7. Configuration for slepton trapping in gravitino superWIMP scenarios 33 .

At the same time, because some sleptons will be slowly moving and highly-ionizing, they may be trapped and studied [33, 34, 35, 36]. As an example, sleptons may be trapped in water tanks placed outside collider detectors. These water tanks may then be drained periodically to underground reservoirs where slepton decays can be observed in quiet environments. This possibility has been studied in Ref. [33] and is illustrated in Fig. 7. The number of sleptons that may be trapped is model-dependent, but may be as large as thousands per year.

If thousands of sleptons are trapped, the slepton lifetime may be determined to the few percent level simply by counting the number of slepton decays as a function of time. The slepton mass will be constrained by analysis of the collider event kinematics. Furthermore, the outgoing lepton energy can be measured, and this provides a high precision measurement of the gravitino mass, and therefore a determination of the gravitino relic density through Eq. (6). As with the case of WIMPs, consistency at the percent level with the observed dark matter relic density will provide strong evidence that dark matter is indeed composed of gravitino superWIMPs.

Perhaps as interesting, the determination of $\tau, m_{\tilde{G}}$, and $m_{\tilde{\tau}}$ in Eq. (7) implies that one can determine Newton's constant on the scale of fundamental particles [37, 38]. According to conventional wisdom, particle colliders are insensitive to gravity, since it is such a weak force. We see that this is not true - if $G_{N}$ enters in a decay time, one can achieve the desired sensitivity simply by waiting a long time. In this case, one can measure the force of gravity between two test particles with masses $\sim 10^{-27} \mathrm{~kg}$, a regime that has never before been probed. If this force is consistent with gravity, these studies will show that the newly discovered particle is indeed interacting gravitationally, as is required for the gravitino to be the graviton's superpartner, and demonstrate that gravity is in fact extended to supergravity in nature. 
As noted above, gravitinos are not the only known superWIMPs - other examples include axinos [25], quintessinos [26], and Kaluza-Klein graviton and axion states [27]. These have interactions that differ from gravitinos either slightly or drastically, and another implication of the measurements just described will be that they will be able to distinguish these possibilities, and likely exclude many and favor one.

The identification of superWIMP dark matter will have many astrophysical implications. Decays that produce superWIMPs also typically release electromagnetic and hadronic energy. This energy may modify the light element abundances predicted by standard $\mathrm{BBN}$ or distort the black body spectrum of the CMB [21]. In addition, superWIMP dark matter may behave as warm dark matter [39, 40, 41, 42, 43] in contrast to WIMPs. Collider studies will therefore provide a window on the early universe, with important consequences for structure formation and other topics.

\section{Dark Energy}

Recent observations of dark energy provide profound problems for particle physics. In quantum mechanics, an oscillator has zero-point energy $\frac{1}{2} \hbar \omega$. In quantum field theory, the vacuum energy receives contributions of this size from each mode, and so is expected to be $\rho_{\Lambda} \sim \int^{E} d^{3} k \frac{1}{2} \hbar \omega \sim E^{4}$, where $E$ is the energy scale up to which the theory is valid. Typical expectations for $\rho_{\Lambda}^{1 / 4}$ are therefore the weak scale or higher, whereas the observed value is $\rho_{\Lambda}^{1 / 4} \sim \mathrm{meV}$. This discrepancy is the cosmological constant problem. Its difficulty stems from the fact that the natural energy scale for solutions is not at high energies yet to be explored, but at low energies that one would otherwise have assumed are well-understood.

Can upcoming colliders provide any insights? It would be pure fancy at this stage to propose an experimental program to solve the cosmological constant problem. On the other hand, the possibility of probing very early times in the Universe's history implies that one may be sensitive to an era when the effects of dark energy were amplified relative to the present.

As an example, assume that the Friedmann equation takes the form

$$
H^{2}=\frac{8 \pi G_{N}}{3}(\rho+\Delta \rho)
$$

where $\Delta \rho$ is a new, exotic contribution to the energy density. This modification to the Hubble parameter directly impacts the evolution of the dark matter density $n$ through its presence in the Boltzmann equation

$$
\frac{d n}{d t}=-3 H n-\left\langle\sigma_{A} v\right\rangle\left(n^{2}-n_{\mathrm{eq}}^{2}\right) .
$$

If one determines the dark matter thermal relic density as outlined in Sec. 3.2, one therefore simultaneously bounds contributions $\Delta \rho$ to $H$.

This approach has been explored in a few recent studies [44, 45, 46]. In Ref. [46], the exotic energy density contributions are assumed to be of the form $\Delta \rho \propto T^{n}$. For various values of $n$ between 0 and 8 , this parametrization can accommodate a wide variety 


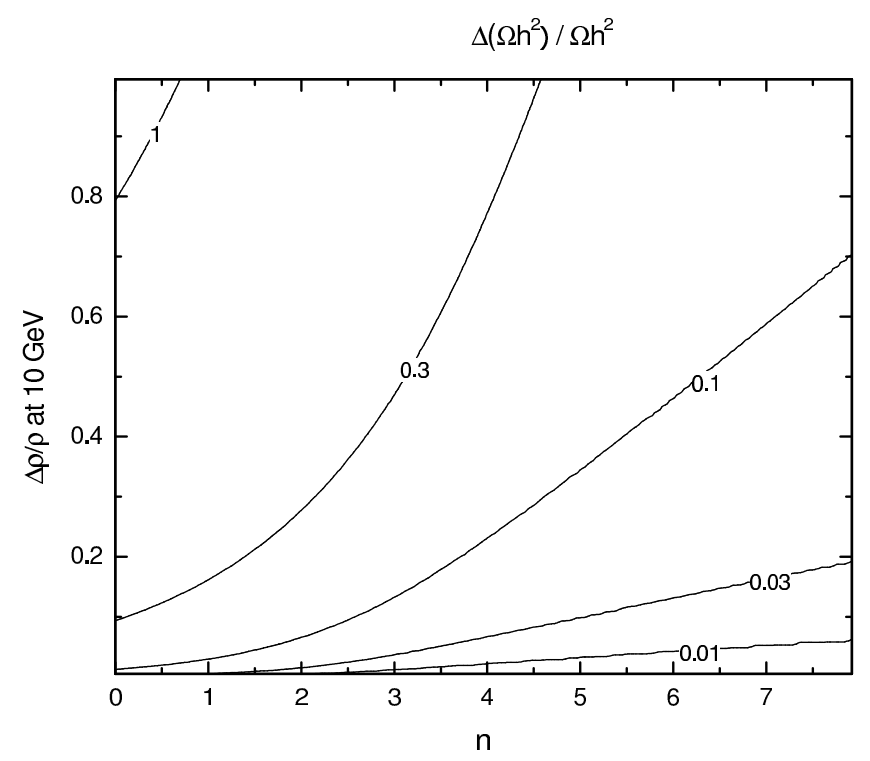

Figure 8. The fractional change in thermal relic density $\Delta\left(\Omega_{\chi} h^{2}\right) /\left(\Omega_{\chi} h^{2}\right)$ for exotic contributions $\Delta \rho \propto T^{n}$, as a function of $n$ and $\Delta \rho / \rho$ at $T=10 \mathrm{GeV}$ [46].

of possibilities, including, for example, a cosmological constant, quintessence, tracking dark energy, and variations in $G_{N}$. The results are given in Fig. 8, Not surprisingly, the thermal relic density is a sensitive probe of new contributions to dark energy, provided that they are significant at the time of freeze out. For example, for $n=4$, a collider measurement that bounds the particle physics prediction for the thermal relic density $\Omega_{\chi} h^{2}$ with a fractional uncertainty of $10 \%$ also bounds variations in the energy density of the order of $10 \%$ at temperatures $\sim 10 \mathrm{GeV}$. This provides a constraint on variations in $G_{N}$ in the very early universe. Alternatively, these results could favor some proposals for dark energy and exclude others.

\section{Conclusions}

In the coming year, the LHC will probe the weak scale $M_{\text {weak }} \sim 100 \mathrm{GeV}-1 \mathrm{TeV}$ in great detail. This has implications for new particle physics, but may also open up new windows on the early universe, and tests of gravity in rather unusual regimes.

At present, the evidence for particle dark matter is as strong as ever. The possibility of WIMP dark matter is well-motivated, and there has been a recent proliferation of candidates. At the same time, there has also been a great deal of progress on the alternative possibility of superWIMP dark matter. In virtually all cases, the LHC will be able to produce these candidates, and in some cases, precision measurements at the LHC may be able to determine the candidate's relic density. Comparison with observations may then provide compelling evidence that particles produced at the LHC do, in fact, constitute the dark matter. Such studies will also be able to determine the microscopic properties of the WIMP particle. 
Colliders may also provide an interesting window on gravity in unusual environments. For example, in the superWIMP scenarios, one may probe gravitational interactions between fundamental particles and provide quantitative evidence for supergravity. In the WIMP scenarios, the thermal relic density studies simultaneously bound new contributions to dark energy at the time of freezeout, probing variations in the strength of gravity at $\sim 1$ ns after the Big Bang, and possibly shedding light on the dark energy problem.

It is rather striking that in many of these scenarios, the LHC, along with other observatories and experiments, could solve many old questions, such as the identity and origin of dark matter. If any of the ideas discussed here is realized in nature, the interplay of collider physics with cosmology and astrophysics in the next few years will likely yield profound insights about the Universe, its contents, and its evolution.

\section{Acknowledgments}

I thank the organizers of GRG18/Amaldi7 for the invitation to participate in this stimulating conference and my collaborators for their many insights regarding the work discussed here. This work was supported in part by NSF Grants PHY-0239817 and PHY-0653656, NASA Grant NNG05GG44G, and the Alfred P. Sloan Foundation.

\section{References}

[1] D. N. Spergel et al. [WMAP Collaboration], Astrophys. J. Suppl. 170, 377 (2007) arXiv:astro-ph/0603449.

[2] M. Tegmark et al., Phys. Rev. D 74, 123507 (2006) arXiv:astro-ph/0608632.

[3] R. A. Knop et al. [The Supernova Cosmology Project Collaboration], Astrophys. J. 598, 102 (2003) arXiv:astro-ph/0309368.

[4] J. Wudka, arXiv:hep-ph/0307339

[5] H. C. Cheng and I. Low, JHEP 0309, 051 (2003) arXiv:hep-ph/0308199].

[6] G. Jungman, M. Kamionkowski and K. Griest, Phys. Rept. 267, 195 (1996) arXiv:hep-ph/9506380.

[7] HEPAP LHC/ILC Subpanel, "Discovering the Quantum Universe," http://www.linearcollider.org.

[8] H. Goldberg, Phys. Rev. Lett. 50, 1419 (1983).

[9] J. R. Ellis, J. S. Hagelin, D. V. Nanopoulos, K. A. Olive and M. Srednicki, Nucl. Phys. B 238, 453 (1984).

[10] G. Servant and T. M. P. Tait, Nucl. Phys. B 650, 391 (2003) arXiv:hep-ph/0206071].

[11] H. C. P. Cheng, J. L. Feng and K. T. Matchev, Phys. Rev. Lett. 89, 211301 (2002) arXiv:hep-ph/0207125.

[12] J. A. R. Cembranos, A. Dobado and A. L. Maroto, Phys. Rev. Lett. 90, 241301 (2003) arXiv:hep-ph/0302041.

[13] J. A. R. Cembranos, A. Dobado and A. L. Maroto, Phys. Rev. D 68, 103505 (2003) arXiv:hep-ph/0307062.

[14] See, e.g., ATLAS Detector and Physics Performance Technical Design Report, http://atlas.web.cern.ch/Atlas/GROUPS/PHYSICS/TDR/access.html, CMS Physics Technical Design Report, http://cmsdoc.cern.ch/cms/cpt/tdr, and references therein.

[15] T. Appelquist, H. C. Cheng and B. A. Dobrescu, Phys. Rev. D 64, 035002 (2001) arXiv:hep-ph/0012100. 
[16] B. C. Allanach, G. Belanger, F. Boudjema and A. Pukhov, JHEP 0412, 020 (2004) arXiv:hep-ph/0410091.

[17] T. Moroi, Y. Shimizu and A. Yotsuyanagi, Phys. Lett. B 625, 79 (2005) arXiv:hep-ph/0505252.

[18] A. Birkedal et al., arXiv:hep-ph/0507214.

[19] E. A. Baltz, M. Battaglia, M. E. Peskin and T. Wizansky, Phys. Rev. D 74, 103521 (2006) arXiv:hep-ph/0602187.

[20] Report of the Cosmology Subgroup, American Linear Collider Physics Group, summarized in J. L. Feng, In the Proceedings of 2005 International Linear Collider Workshop (LCWS 2005), Stanford, California, 18-22 Mar 2005, pp 0013 arXiv:hep-ph/0509309; J. Phys. G 32, R1 (2006) arXiv:astro-ph/0511043.

[21] J. L. Feng, A. Rajaraman and F. Takayama, Phys. Rev. Lett. 91, 011302 (2003) arXiv:hep-ph/0302215; Phys. Rev. D 68, 063504 (2003) arXiv:hep-ph/0306024.

[22] J. R. Ellis, K. A. Olive, Y. Santoso and V. C. Spanos, Phys. Lett. B 588, 7 (2004) arXiv:hep-ph/0312262.

[23] J. L. Feng, S. Su and F. Takayama, Phys. Rev. D 70, 063514 (2004) arXiv:hep-ph/0404198; Phys. Rev. D 70, 075019 (2004) arXiv:hep-ph/0404231.

[24] L. Roszkowski and R. Ruiz de Austri, JHEP 0508, 080 (2005) arXiv:hep-ph/0408227.

[25] L. Covi, J. E. Kim and L. Roszkowski, Phys. Rev. Lett. 82, 4180 (1999) arXiv:hep-ph/9905212; L. Covi, H. B. Kim, J. E. Kim and L. Roszkowski, JHEP 0105, 033 (2001) arXiv:hep-ph/0101009.

[26] X. J. Bi, M. z. Li and X. m. Zhang, Phys. Rev. D 69, 123521 (2004) arXiv:hep-ph/0308218.

[27] J. L. Feng, A. Rajaraman and F. Takayama, Phys. Rev. D 68, 085018 (2003) arXiv:hep-ph/0307375.

[28] R. Kitano and I. Low, arXiv:hep-ph/0503112.

[29] M. Drees and X. Tata, Phys. Lett. B 252, 695 (1990).

[30] J. L. Goity, W. J. Kossler and M. Sher, Phys. Rev. D 48, 5437 (1993) arXiv:hep-ph/9305244.

[31] A. Nisati, S. Petrarca and G. Salvini, Mod. Phys. Lett. A 12, 2213 (1997) arXiv:hep-ph/9707376.

[32] J. L. Feng and T. Moroi, Phys. Rev. D 58, 035001 (1998) arXiv:hep-ph/9712499.

[33] J. L. Feng and B. T. Smith, Phys. Rev. D 71, 015004 (2005) arXiv:hep-ph/0409278.

[34] K. Hamaguchi, Y. Kuno, T. Nakaya and M. M. Nojiri, Phys. Rev. D 70, 115007 (2004) arXiv:hep-ph/0409248.

[35] A. Brandenburg, L. Covi, K. Hamaguchi, L. Roszkowski and F. D. Steffen, Phys. Lett. B 617, 99 (2005) arXiv:hep-ph/0501287.

[36] A. De Roeck, J. R. Ellis, F. Gianotti, F. Moortgat, K. A. Olive and L. Pape, Eur. Phys. J. C 49, 1041 (2007) arXiv:hep-ph/0508198.

[37] W. Buchmuller, K. Hamaguchi, M. Ratz and T. Yanagida, Phys. Lett. B 588, 90 (2004) arXiv:hep-ph/0402179.

[38] J. L. Feng, A. Rajaraman and F. Takayama, Int. J. Mod. Phys. D 13, 2355 (2004) arXiv:hep-th/0405248.

[39] K. Sigurdson and M. Kamionkowski, Phys. Rev. Lett. 92, 171302 (2004) arXiv:astro-ph/0311486.

[40] S. Profumo, K. Sigurdson, P. Ullio and M. Kamionkowski, Phys. Rev. D 71, 023518 (2005) arXiv:astro-ph/0410714.

[41] M. Kaplinghat, Phys. Rev. D 72, 063510 (2005) arXiv:astro-ph/0507300.

[42] J. A. R. Cembranos, J. L. Feng, A. Rajaraman and F. Takayama, Phys. Rev. Lett. 95, 181301 (2005) arXiv:hep-ph/0507150.

[43] K. Jedamzik, M. Lemoine and G. Moultaka, JCAP 0607, 010 (2006) arXiv:astro-ph/0508141.

[44] M. Drees, H. Iminniyaz and M. Kakizaki, Phys. Rev. D 76, 103524 (2007) arXiv:0704.1590 [hep$\mathrm{ph}]]$.

[45] D. J. H. Chung, L. L. Everett, K. Kong and K. T. Matchev, JHEP 0710, 016 (2007) arXiv:0706.2375 [hep-ph]].

[46] S. M. Carroll, J. L. Feng, D. W. Hsu, in preparation. 